\title{
LA ESQUINA DEL FUTURO: ESCRIBIR ENTRE EL BARRIO Y LA ESCUELA
}

\author{
Mariano Dubin \\ Facultad de Humanidades y Ciencias de la Educación \\ Instituto de Investigaciones en Humanidades y Ciencias Sociales \\ Universidad Nacional de La Plata \\ Consejo Nacional de Investigaciones Científicas y Técnicas
}

Resumen: Distintos autores (Chartier, 1995, 1999; Chartier y Cavallo, 1998; Ginzburg, 2008; Sawaya, 2009) han señalado que las formas de leer corresponden a variaciones históricas y sociales. Considerando esta perspectiva, a partir de ciertos modos de la cultura barrial que se presentan en la escritura de alumnos en clases de lengua $y$ literatura indagamos los marcos interpretativos de las lecturas de los jóvenes de la periferia urbana.

Palabras clave: Cultura popular, cultura escolar, escritura, literatura, barrios de la periferia.

\begin{abstract}
Several authors (Chartier, 1995, 1999; Chartier y Cavallo, 1998; Ginzburg, 2008; Sawaya, 2009) have stated that the ways of reading are plural and diverse and, moreover, they are related to historical and social variations. Regarding this point of view, the current article analyses ways of working-class neighbourhood culture which appear in secondary school students' writing in the context of literature and language lessons and how it is useful for us as researchers to understand the teeneagers' reading interpretations.
\end{abstract}

Keywords: Popular culture, scholar culture, writing, literature, workingclass neighbourhoods. 
Algunas de las perspectivas críticas que han analizado la lectura a partir de sus claves sociales, culturales e históricas (Chartier, 1995, 1999; Chartier y Cavallo, 1998; Ginzburg, 2008; Sawaya, 2009) nos han advertido de la operación ideológica que dispone que los modos y situaciones de lectura propios de las clases sociales hegemónicas sean los naturales, los únicos o los válidos. Desplazarnos de una idea abstracta de lectura hacia un análisis situado de las prácticas de lectura nos permite ingresar a un complejo mundo de lectores y lecturas efectivas que se enmarcan en distintos tipos de relaciones sociales caracterizadas por el conflicto y la diversidad.

La heterogeneidad social, cultural y lingüística que constituye a cada sociedad, y que se pone en juego en las lecturas particulares de los sujetos, se presenta como un elemento a considerar en el análisis que introduciremos sobre modos de las culturas barriales en la escritura escolar de jóvenes de la periferia urbana. La Escuela ha establecido históricamente ciertas prácticas como las legítimas sin atender la diversidad o imponiendo unas prácticas sobre otras (Cuesta, 2011; Lahire, 2008; Rockwell, 2000, 2011; Sawaya, 2009), principalmente, como hemos indicado, desde una clave clasista: son los modos y las situaciones (de lectura) de las clases populares los que la norma escolar negativiza. Esta tensión, que en nuestro caso podríamos presentar en el cruce entre la cultura escolar y la cultura barrial, se tornaría más evidente (y más conflictiva) en las clases de lengua y literatura ya que, justamente, parte de la asignatura consiste en la enseñanza de la lectura e interpretación de textos 
introduciendo (siendo o no habilitadas) las voces de los alumnos.

Las interpretaciones de los alumnos nos permiten ingresar a unos recorridos de lecturas, concepciones de mundo, posicionamientos artísticos e ideológicos, etc., que no son necesariamente compartidos (o siquiera legitimados) por las tradiciones de enseñanza escolar. Carolina Cuesta (2006, 2010, 2011, 2013) ha analizado cómo ciertos saberes del orden social y cultural, que operan en las intervenciones de los alumnos, no convalidan lo que ciertas teorías -como el estructuralismo y sus actualizaciones en la llamada nueva narratología- han establecido como las respuestas esperables ${ }^{1}$. Este caso, como otros de orden diverso, suelen ser explicados en términos de carencia de los alumnos.

Por el contrario, nos proponemos avanzar en un estudio sobre las lecturas de los alumnos -en tanto marcos interpretativos- recortando como material de análisis sus escritos escolares. De tal modo, introduciremos un escrito llamado "La esquina del futuro" que fue producido por dos alumnos en un cuarto año de la materia Literatura, en la Escuela Media No1 de la ciudad de Berisso, de un curso de

1 La narratología ha considerado a la narración como aparato formal, es decir, sus componentes serían susceptibles de ser deslindados y explicados en particular (Bal, 1998; Genette, 1989). Los alumnos al hacer ingresar nociones provenientes de sus concepciones de mundo para interpretar los textos -recuperando, por ejemplo, posicionamientos biográficos o morales- contrastan con los modos de enseñanza que analizan la literatura por fuera de sus consideraciones históricas y autorales (Cuesta, 2010 , p. 8). Cuesta (2013) propone revisar las nociones de experiencia para recuperar las maneras en que los alumnos leen literatura en relación a un hablar de la vida, sujeto a distintos órdenes de lo real y regímenes de verdad (2013, pp. 116 - 117).

№3. Primer Semestre de 2015 
quince alumnos. Su elección se debe a que ejemplifica una recurrencia de nuestro corpus ${ }^{2}$ : una escritura que no sólo refiere al barrio sino que se organiza a partir de una voz barrial.

\section{El barrio como un modo narrativo}

Las clases populares poseen una diversidad de clases y grupos sociales en su conformación histórica (Gramsci, 1974, p. 82) que requieren para su abordaje un análisis situado que dé cuenta de su complejidad. Los desarrollos clásicos sobre clase obrera, clases populares y subalternas (Gramsci, 1992; Lenin, 2013; Lukács, 2009; Marx, 2000; Thompson, 1989) son útiles para establecer, en términos generales, procesos históricos de orden global pero exigen una revisión constante y la construcción de nuevas categorías para indagar los problemas particulares de las clases populares en América Latina y, específicamente, en Argentina (Adamovsky, 2012; Di Meglio, 2011). Nos interesa articular una serie de teorías que han ido conformando diversos campos de estudio sobre las clases y las culturas populares con una opción metodológica etnográfica que busque comprender los fenómenos sociales desde la perspectiva de sus miembros (Guber, 2006, 2008).

2 El corpus ha sido recolectado a partir del año 2005 en contextos de educación formal y no formal en barrios de la periferia de la ciudad de La Plata, donde me he desempeñado como docente (Dubin y Provenzano, 2010; Dubin, 2011). Asimismo, este corpus ha sido enriquecido con la lectura y análisis de escritos de alumnos producidos en diversos contextos educativos que he ido conociendo a través del trabajo de colegas y la lectura regular de registros escolares en mis funciones de docencia e investigación en la Universidad Nacional de La Plata y la Universidad Pedagógica. 
En relación a los estudios sobre culturas populares, estos han experimentado un cambio de enfoque de unas teorías que las pensaban como versiones degradadas de la "alta cultura" hacia análisis que han señalado su carácter autónomo y/o contrahegemónico. Ha sido, posiblemente, La cultura popular en la Edad Media y en el Renacimiento: El contexto de François Rabelais [1965] (1988) de Mijail Bajtin, uno de los textos que sustanció este cambio epistemológico.

Bajtin no sólo establece la complejidad de su estudio -un mundo donde el cuerpo, los espacios públicos, la transgresión a la norma oficial, el humor irreverente, las concepciones regeneradoras y materialistas del universo corresponden a una historia particular y milenaria- sino que complejiza el análisis formal de los textos literarios introduciendo una clave de lectura: Gargantúa y Pantagruel de François Rabelais sólo puede ser entendido cabalmente recuperando los sentidos propios de las tradiciones populares europeas ${ }^{3}$.

Sin poder profundizar en el presente trabajo el marco teórico y metodológico enunciado, sostenemos como punto de

3 El desarrollo de Bajtin es mucho más vasto y complejo al atendido en esta instancia donde nos interesa abordar la apropiación espacial por las clases populares. Señalemos, de manera somera, que Bajtin entiende a la cultura popular como una cultura cómica popular basada en concepciones cíclicas de muerte y nacimiento que borra las fronteras clásicas del sujeto. A diferencia del cuerpo clásico que se presenta perfectamente acabado, sin mezcla, individual y expresivo, el cuerpo grotesco no está separado del resto del mundo ni es perfecto, sino que sale fuera de sí, franquea sus propios límites (1988, p. 37). Esta cultura toma lugar principal durante el carnaval y otras fiestas populares donde irrumpe una visión no oficial que parodia los ritos y usos de la cultura oficial que constituyen una dualidad en la percepción del mundo y la vida humana (1988, p. 11). 
partida que una de las maneras de llevar a cabo un análisis situado de las culturas populares es considerar su ocupación del espacio urbano. Siguiendo a Bajtin (1988), repensamos el caso de una cultura popular rioplatense recortando al barrio como su espacio privilegiado. Un espacio que no sólo remite a un lugar sino, principalmente, a una manera de circularlo 4 .

Citemos el escrito objeto de nuestro trabajo realizado por los alumnos Joel y Andrés. Se titula "La esquina del futuro":

En la esquina del futuro hay un robot con un parlante grande que suena fuerte, que tiene entrada USB, y en la panza una heladera para el "tang" (una bebida especial) que tiene un lugar para guardar el medicamento por si alguien se siente mal...

Después de tomar un juguito y el medicamento por si les duele la cabeza en el baile, salen los pibes de la esquina (Rulo, Durito, Negro, Morza, el Pata y el robot “el chapa”).

En el baile lo mandan al Chapa a chamuyar a la pibita que le gusta al Durito, total si lo corta no tiene el sentimiento de la vergüenza, pero si sale el chamuyo va el durito. Y si pinta el bondi el Chapa va al frente y si se come alguna no importa porque no le duele, le duele al otro.

A la salida del baile iban pateando para el barrio y se cruzaron con otra banda que tenían otro robot pero no era nada al lado del chapa y este quería boxiar y el chapa le

4 Las nociones de barrio y cultura barrial exigen un análisis más detenido que el que, en esta instancia, podemos desarrollar y se extiende a una diversidad de perspectivas y debates sobre la definición del espacio urbano y sus maneras de circulación social y cultural. Ver: de Certeau (2006-2007), Chaves (2010), García Canclini (2005), Gorelik (1998), Reguillo-Cruz (1991). 
ganó, le sacó la heladera y todo el medicamento para el dolor de cabeza y la otra banda se fueron sin robot. Guardaron para el otro día el juguito tang y el medicamento en el chapa y se quedaron escuchando música hasta que cantó el gallo.

En clase se había leído en voz alta el cuento "Marionetas S.A" de Ray Bradbury. Al finalizar la lectura, comenzó un intercambio entre el docente y los alumnos. Parte de los alumnos expresaron que no concordaban con la visión aparentemente negativa que se presentaba de los robots, quienes reemplazaban a los humanos en ciertas actividades que estos preferían no realizar. Durante la lectura, algunos de los comentarios registrados fueron: "con uno así no voy más a la escuela", "lo dejo en casa así mi vieja piensa que estoy estudiando y estoy de joda en el boliche", "así la re cuerneo a mi novia" y la réplica de Evelyn a este último: "así seguro que te cuernean a vos". Esta alumna comentó, asimismo, que el cuento le había parecido una telenovela y por eso, el final, no la sorprendió. Durante el intercambio se propuso, oralmente, escribir un cuento de ciencia ficción situado en Argentina. Joel y Andrés escribieron, entonces, "La esquina del futuro".

Corrámonos de las representaciones más negativas sobre la esquina. Mariana Chaves (2005) ha analizado cómo en los discursos hegemónicos (a través de diarios, revistas, programas radiales y televisivos, entre otros) se construye al joven como un actor social sin capacidad de acción. Estas representaciones se instituyen, de hecho, como un obstáculo epistemológico para el conocimiento del otro (2005, p. 19). Recuperemos, por el contrario, algunas voces de los jóvenes de la periferia al 
representar la esquina y otros espacios barriales. En este caso, Joel y Andrés no encuentran que la esquina sea un espacio negativo. Allí comienzan y cierran la narración: es la cifra a través de la cual caminan por el barrio, van al baile y vuelven al mismo punto.

La esquina no es, como a veces se la suele presentar, un acontecimiento contemporáneo. Ha sido un espacio de encuentro desde el origen de los barrios rioplatenses tal como documenta parte de la poesía, la música y la literatura argentina desde fines del siglo XIX. Sin poder ser exhaustivos, señalemos que en los últimos cien años distintas manifestaciones musicales, que podemos sintetizar desde los primeros tangos de Ángel Villoldo hasta las actuales letras de cumbia villera, han centrado su poética no sólo en tópicos barriales sino que han utilizado el registro popular del habla para dar letra a su música. Pero decir eso sería hacer un recorte que nos aleja ciertamente de la totalidad de la producción social de sentido y de la representación del mundo (Angenot, 2010, p. 17). Estamos pensando el discurso social en los términos de Marc Angenot que lo inscribe en los sistemas genéricos, los repertorios tópicos, las reglas de encadenamiento de enunciados que, en una sociedad dada, organiza lo decible lo narrable y lo opinable- y aseguran la división del trabajo discursivo (2010, p. 21).

Si sorteamos los recortes más exiguos de los análisis formales anclados en fuertes tradiciones disciplinares, podríamos pensar el lugar del barrio y de la esquina en una concatenación mayor de discursos que recupera el tema barrial entramado en el habla popular: desde el Jorge Luis Borges de 
"Hombre de la esquina rosada", las aguafuertes de Roberto Arlt y, previamente, las crónicas periodísticas de Soyza Reilly y Last Reason, la poesía de Enrique Santos Discépolo y los poetas del tango de la década del '20 y el '30, el teatro de Alberto Vacarezza, los poetas lunfardescos, las novelas de Joaquín Gómez Bas, pasando por algunos cuentos de Bernardo Kordon, Haroldo Conti y Roberto Fontanarrosa y Flores robadas en los jardines de Quilmes de Jorge Asís hasta la serie Okupas y el nuevo cine argentino, la poesía de Washington Cucurto y Oscar Fariña, Kryptonita de Leonardo Oyola, la letrística de Damas Gratis, Pibes Chorros y Meta Guacha, La virgen cabeza de Gabriela Cabezón Cámara, Corte Rancho de César González o la revista La garganta poderosa para nombrar sólo algunas producciones artísticas más o menos conocidas, pero que conviven con otras producciones menos prestigiosas -pero igual de determinantes desde esta perspectiva discursivatal como el periodismo deportivo, la tradición del humor nacional, las propagandas de productos populares, los diarios sensacionalistas y algunas producciones teatrales y televisivas masivas. A su vez, este repertorio tópico no aparece sólo en las producciones discursivas de circulación pública y masiva sino en los usos cotidianos: en la charla del bar, en la mesa familiar, en la discusión política, etc.

La esquina de "La esquina del futuro" opera en una serie que puede recordarnos de manera más inmediata a la letrística de la cumbia villera pero que se extiende a campos de sentidos más amplios pero relacionados en una totalidad interdiscursiva que conforma lo que Angenot refiere como "rumor social" (2010, p. 23). A través de una operación radical de 
desclausuramiento de los campos discursivos tradicionalmente investigados como si existieran aislados y fueran autónomos en la totalidad de lo que se imprime y se enuncia (2010, p. 22) podríamos relevar el tópico de la esquina en discursos de procedencias históricas, sociales y culturales diversas.

Evaristo Carriego, por ejemplo, en Misas Herejes (1908), había escrito sobre la esquina como reunión de amigos: "La esquina o el patio, de alegres reuniones, / le oye contar hechos, que nadie le niega: / ¡con una guitarra de altivas canciones / él es Juan Moreira, y él es Santos Vega! ("El guapo"). En una novela reciente, Kryptonita (2011) de Leonardo Oyola, aparece la esquina y la juntada de barrio en las historias de un grupo de superhéroes en versiones arrabaleras -la novela supone qué hubiera sucedido si Superman en vez de caer en Smallville (Kansas) desde su planeta Krypton hubiera caído en Los Eucaliptos, una villa de La Matanza-. ¿Pero qué podríamos encontrar en común en las esquinas de Evaristo Carriego, de Leonardo Oyola y de Joel y Andrés? La esquina se presenta, con sus variaciones, como el lugar donde uno se curte: es una conocimiento que exige poner el cuerpo y, a su vez, la esquina como lugar de pertenencia, convierte a los participantes en iniciados donde es central compartir ciertos usos de la lengua como índice de haber vivido, y a veces sobrevivido, a ciertas experiencias.

La teórica paulista Sandra Sawaya, en sus trabajos sobre las prácticas de lectura en barrios de la periferia de San Pablo, ha utilizado el concepto de "cartografiar el barrio" para señalar un modo narrativo característico de niños y jóvenes de barrios de la periferia: 
Construir la historia del barrio, cartografiarlo, es otra de las funciones de la palabra para los niños, lo que termina transformándolos en portavoces, en memoria viva $\mathrm{y}$ colectiva de la vida del barrio y de las personas que en él viven: son informantes, palomas mensajeras, delatores y testigos (...) Las historias contadas por los niños sobre el barrio y las familias trazan el recorrido existencial de la vida de aquellas personas, situándolas en el espacio y en el tiempo (Sawaya, 2009, p. 59).

Recuperamos el concepto de cartografiar el barrio para ir más allá de un análisis formal de "La esquina del futuro" y analizar la apropiación que se hace del género de ciencia ficción desde la experiencia de estos jóvenes y sus maneras de sentir y explicar el mundo. En tal sentido, muchos jóvenes, en clases de literatura, traen a consideración sentidos de la cotidianeidad barrial para interpretar (y de tal modo reescribir) los textos literarios.

Un fenómeno que podríamos relacionar a lo que Denis Merklen (2005) considera una de las características principales de las clases populares argentinas: su inscripción territorial. Pero entendamos, en el caso analizado, la inscripción territorial no sólo referida a ciertos tópicos sino, principalmente, como una forma. En "La esquina del futuro" el barrio es un modo de narrar construido en su propia voz. Una escritura que es atravesada por la memoria colectiva del barrio y donde quedan las huellas de la experiencia en las orillas contemporáneas. 


\section{Disputas por el lugar de la interpretación}

Edward Said había destacado en Cultura e Imperialismo (1994, p. 48), retomando a Gramsci, que la hegemonía burguesa se establece como una forma de organizar lo geográfico; no sólo en una organización material - económica sino, principalmente, simbólica. El discurso hegemónico borra deliberadamente ciertos espacios. Zygmunt Bauman los llamó "espacios vacíos" (2004): ciertos lugares (como algunos barrios obreros) que son eliminados del mapa de los imaginarios sociales dominantes. En tal sentido, cuando las diversas manifestaciones de las culturas subalternas remiten a sus lugares de pertenencia no podemos entenderlos como actos ingenuos sino que deben ser inscriptos en la continua conflictividad entre distintos discursos y grupos sociales en torno al eje centro - periferia (Chaves, 2005, 2011, 2012).

La cotidianidad escolar, entramada en los discursos que la atraviesan, prolonga esta conflictividad tal como hace notar Mariana Provenzano (2015) al señalar cómo en algunos escritos de alumnos pertenecientes a jóvenes de la periferia urbana se presentan las tensiones entre las representaciones altamente negativizadas de los discursos hegemónicos y las propias maneras de representarse a sí mismos, sus barrios y sus prácticas cotidianas. Es posible que los modos narrativos de corte barrial -y, a su vez, una serie de expresiones barriales que se descubren en el aula y en otros espacios escolares- tense algunas prácticas docentes. De cierto modo, esta tensión es constitutiva: la cultura escolar existe atravesada por las 
contradicciones existentes. No existe una enseñanza que esté escindida de los distintos conflictos sociales.

\section{A modo de conclusión}

Si bien asumimos las hipótesis previas como provisorias, y señalamos que la presente investigación deberá revisar algunas de las nociones utilizadas para lograr un análisis más preciso sobre las culturas barriales y populares contemporáneas, podemos afirmar que defender la escritura de jóvenes de barrios de la periferia en su complejidad nos alienta a transitar el difícil y necesario ejercicio, como docentes e investigadores, de conocer el mundo escolar desde la perspectiva de los alumnos.

Si asumimos que los alumnos se relacionan con la literatura poniendo en juego modos de lectura específicos (Cuesta, 2006) que no implican sólo formas de leer literatura sino, fundamentalmente, formas de leer la vida (Sawaya, 2009), esta investigación exige, necesariamente, como punto de partida, una tarea de documentación de las diversas producciones de los alumnos que han sido invisibilizadas en la cultura escolar, es decir, realizar una tarea de documentar lo no documentado (Ezpeleta y Rockwell, 1983; Rockwell, 2011).

La recopilación de escrituras escolares se presenta, actualmente, como una vacancia en la investigación didáctica ${ }^{5}$.

5 Cabe mencionar como una de las excepciones a esta vacancia la recopilación realizada por Malena Botto (2012). 
Dar cuenta de ellas nos posibilitaría ingresar a un mundo social y cultural que ha sido, mayormente, obviado o presupuesto y al que, pocas veces, se le ha dado estatuto de análisis crítico. No buscamos, sin embargo, crear un nuevo objeto de estudio puramente especulativo sino, por el contrario, apelamos a una articulación de los niveles descriptivos y explicativos con desarrollos de problemáticas relacionadas con la inclusión (cómo introducir en las prácticas docentes la perspectiva de los alumnos), con políticas educativas (cómo diseñar estrategias posibles que ataquen al fracaso escolar construido, en parte, desde la creación de estereotipos negativos sobre jóvenes de la periferia urbana) y con propuestas didácticas (cómo repensar la enseñanza de problemas disciplinares y los mismos problemas disciplinares a partir de una cultura escolar situada). 


\section{Bibliografía}

Adamovsky, E. (2012). Historia de las clases populares en la Argentina. Tomo II (Desde 1880 hasta 2003). Buenos Aires: Sudamericana.

Angenot, M. (2010). El discurso social. Los límites de lo pensable y lo decible. Buenos Aires: Siglo XXI.

Bajtin, M. (1988) [1965]. La Cultura Popular en la Edad Media $y$ en el Renacimiento. El contexto de François Rabelais. Madrid: Alianza.

Bal, M. (1998). Teoría de la narrativa. Introducción a la narratología. Madrid: Cátedra.

Bauman, Z. (2004). Modernidad líquida. Buenos Aires: Fondo de Cultura Económica.

Botto, M. (2012). "Los textos literarios de los alumnos". Una investigación cualitativa de las prácticas de lectura y escritura de textos literarios y de las ciencias. Programa de apoyo para la articulación entre la escuela media y los estudios superiores. Buenos Aires: Ministerio de Educación.

Carriego, E. (2013). Obra poética completa. Buenos Aires: Museo Quinquela Martín.

Chartier, R. (1995). Sociedad y escritura en la edad moderna. La cultura como apropiación. México: Instituto Mora.

Chartier, R. (1999). El mundo como representación. Historia cultural: entre práctica y representación. Barcelona: Gedisa.

Chartier, R. y Cavallo, G. (1998). Historia de la lectura en el mundo occidental. Madrid: Taurus.

Chaves, M. (2005). "Juventud negada y negativizada: representaciones y formaciones discursivas vigentes en la Argentina contemporánea". Última Década. Núm. 23: Valparaíso, Diciembre, pp. 9 - 32. 
Chaves, M. (2010). Jóvenes, territorios y complicidades. Buenos Aires: Espacio Editorial.

Chaves, M. (2011). "Jóvenes entre el centro y la periferia de la ciudad, del Estado y de la academia”. J. Carpio. Las políticas sociales urbanas y la construcción de ciudadanía. Buenos Aires: Paidós.

Chaves, M. (2012). "Culturas juveniles en la tapa del diario: tensiones entre el margen y el centro de la hoja". M. Chaves y J. E. Fidalgo Zeballos (Coords.). Políticas de infancia y juventud: producir sujetos, construir Estado, disputar sentidos. Buenos Aires: Espacio-Foro-CIC.

Cuesta, C. (2006). Discutir sentidos. La lectura literaria en la escuela. Buenos Aires: Libros del Zorzal.

Cuesta, C. (2010). "Enseñanza de la literatura: de teorías y lecturas". Leitura, Teoria \& Prática. Revista Semestral de la Asociación de Lectura de Brasil. Año 28, Núm. 55: Campinas, Global Editora, pp. 5 - 12.

Cuesta, C. (2011). Lengua y literatura: disciplina escolar. Hacia una metodología circunstanciada de su enseñanza. Tesis de Doctorado: Facultad de Humanidades y Ciencias de la Educación. Universidad Nacional de La Plata.

Cuesta, C. (2013). "La enseñanza de la literatura y los órdenes de la vida: lectura, experiencia y subjetividad". Literatura: teoría, historia, crítica. Vol. 15. Núm. 2: Bogotá, Universidad Nacional de Colombia, pp. 97 - 119.

de Certeau, M. (2006-2007). La invención de lo cotidiano. II tomos. México: Universidad Iberoamericana.

Di Meglio, G. (2011). Historia de las clases populares en la Argentina. Tomo I (Desde 1516 hasta 1880). Buenos Aires: Sudamericana.

Dubin, M. y Provenzano, M. (2010). "Prácticas de escrituras en espacios de educación no formal". Lulú Coquette. Revista de 
didáctica de lengua y Literatura. Año 2. Núm. 5: Buenos Aires, Ediciones El Hacedor / Jorge Baudino Ediciones, pp. $107-126$.

Dubin, M. (2011). Educación y narrativas en las periferias urbanas. Persistencias y variaciones en las culturas migrantes. Tesis de Licenciatura: Facultad de Humanidades y Ciencias de la Educación. Universidad Nacional de La Plata.

Ezpeleta, J. y Rockwell, E. (1983). La escuela: relato de un proceso de construcción inconcluso. México: Publicaciones del Departamento de Investigaciones Educativas, Centro de Investigación y de Estudios Avanzados del IPN.

García Canclini, N. (2005). Imaginarios urbanos. Buenos Aires: Eudeba.

Genette, G. (1989). Figuras III. Barcelona: Lumen.

Ginzburg, Carlo (2008) [1976]. El queso y los gusanos. El cosmos según un molinero del siglo XVI. Barcelona: Península.

Gorelik, A. (1998). La grilla y el parque. Espacio público y cultura urbana en Buenos Aires, 1887-1936. Quilmes: Universidad Nacional de Quilmes.

Gramsci, A. (1974). Literatura y cultura popular. Tomo I. Buenos Aires: Cuadernos de cultura revolucionaria.

Gramsci, A. (1992) [1929-1935]. Antología. México: Siglo XXI.

Guber, R. (2006). La etnografía. Método, campo y reflexividad. Buenos Aires: Norma.

Guber, R. (2008). El salvaje metropolitano. Reconstrucción del conocimiento social en el trabajo de campo. Buenos Aires: Paidós.

Lahire, B. (2008). “'Iletrismo' y usos sociales de la escritura”. J. Vaca Uribe (Comp.). El campo de la lectura. Veracruz: Universidad Veracruzana. 
Lenin, V. I. (2013) [1918]. El Estado y la revolución. La teoría marxista del Estado y las tareas del proletariado en la revolución. Buenos Aires: Ediciones IPS.

Lukács, G. (2009) [1923]. Historia y conciencia de clase: estudios de dialéctica marxista. Buenos Aires: Razón y Revolución.

Marx, K. (2000) [1867]. El Capital. Tomo I. Madrid: Akal.

Merklen, D. (2005). Pobres ciudadanos, las clases populares en la era democrática (Argentina, 1983-2003). Buenos Aires: Gorla.

Miguez, D. y Semán, P. (2006). Entre santos, cumbias y piquetes: las culturas populares en la Argentina reciente. Buenos Aires: Biblos.

Provenzano, M. (2015). "Aproximaciones a escritos ficcionales en clases de literatura”. M. Papalardo (Comp.). Ingresos a las carreras de Geografia y Letras. Investigaciones sobre prácticas de lectura y escritura. Centro de Investigaciones Geográficas - Facultad de Humanidades y Ciencias de la Educación: En prensa.

Reguillo Cruz, R. (1991). En la calle otra vez. Las bandas: identidad urbana y usos de la comunicación. México: Instituto Tecnológico y de Estudios Superiores de Occidente.

Rockwell, E. (2000). "La otra diversidad: historias múltiples de apropiación de la escritura". DiversCité Langues. Revue et forums interdisciplinaires sur la dynamique des langues. Vol. V: Montréal (Québec).

Rockwell, E. (2011). La experiencia etnográfica. Buenos Aires: Paidós.

Said, E. (1994). Culture and Imperialism. New York: Vintage Books.

Sawaya, S. (2009). "Alfabetización y fracaso escolar: problematizando algunas presuposiciones de la concepción 
constructivista". Lulú Coquette. Revista de didáctica de la lengua y la literatura. Año 3. Número 4: Buenos Aires, Ediciones El Hacedor / Jorge Baudino Ediciones, pp. 53 - 74.

Thompson, E. (1989) [1963]. La formación de la clase obrera en Inglaterra. Barcelona: Crítica.

Fecha de recepción: 1/10/2014

Fecha de aprobación: 24/7/2015 